\title{
Fuzzy soft set theory applied to medical diagnosis using fuzzy arithmetic operations
}

\author{
Yıldıray Çelik* and Sultan Yamak
}

\section{"Correspondence:} ycelik61@gmail.com Department of Mathematics, Karadeniz Technical University, Trabzon, Turkey

\begin{abstract}
In this paper, we apply fuzzy soft set theory through well-known Sanchez's approach for medical diagnosis using fuzzy arithmetic operations and exhibit the technique with a hypothetical case study.
\end{abstract}

AMS Subject Classification: 03E72; 92C50

Keywords: soft set; fuzzy soft set; fuzzy number; defuzzification

\section{Introduction and preliminaries}

Many complicated problems in economics, engineering, social sciences, medical sciences and many other fields involve uncertain data. These problems, which one comes face to face with in life, cannot be solved using classical mathematic methods. In classical mathematics, a mathematical model of an object is devised and the notion of the exact solution of this model is determined. Because of that, the mathematical model is too complex, the exact solution cannot be found. There are several well-known theories to describe uncertainty. For instance, fuzzy set theory [1], rough set theory [2] and other mathematical tools. But all of these theories have their inherit difficulties as pointed out by Molodtsov [3]. To overcome these difficulties, Molodtsov introduced the concept of a soft set as a new mathematical tool for dealing with uncertainties that is free from the difficulties affecting the existing methods. The theory of soft sets has rich potential for applications in several directions, a few of which were demonstrated by Molodtsov in his pioneer work [3]. At present, works on soft set theory are making progress rapidly. Maji et al. [4] initiated the concept of fuzzy soft sets with some properties regarding fuzzy soft union, intersection, complement of a fuzzy soft set, De Morgan's law etc. Neog and Sut [5] have reintroduced the notion of fuzzy soft sets and redefined the complement of a fuzzy soft set accordingly. They have shown that the modified definition of the complement of a fuzzy soft set meets all the requirements that the complement of a set in the classical sense really does. Applications of fuzzy soft set theory in many disciplines and real life situations have been studied by many researchers. De et al. [6] have studied Sanchez's [7, 8] method of medical diagnosis using an intuitionistic fuzzy set. Saikia et al. [9] have extended the method in [6] using intuitionistic fuzzy soft set theory. In [10], Chetia and Das have studied Sanchez's approach of medical diagnosis through IVFSS (interval-valued fuzzy soft set) obtaining an improvement of the same set presented in De et al. [6]. Using the representation of interval-valued fuzzy matrix, Meenakshi and Kaliraja [11] have provided the techniques to study Sanchez's approach of medical diagnosis of interval-valued fuzzy matrices. In 
this paper, by using the notion of a fuzzy soft set together with arithmetic operations on fuzzy number, we apply the fuzzy soft set technology through well-known Sanchez's [8] approach for medical diagnosis, and we exhibit the technique with a hypothetical case study.

In this section, we will give some known and useful definitions and notations regarding a soft set and a fuzzy soft set. The definitions and notions in this part may be found in references $[1,3,4,12-14]$.

Definition $1[3,13]$ Let $U$ be an initial universal set and $E$ be a set of parameters. The power set of $U$ is denoted by $\mathcal{P}(U)$ and $A$ is a subset of $E$. A pair $(F, A)$ is called a soft set over $U$, where $F$ is a mapping given by $F: A \rightarrow \mathcal{P}(U)$.

Definition 2 [1] A fuzzy subset $\mu$ of $U$ is defined as a map from $U$ to [0,1]. The family of all fuzzy subsets of $U$ is denoted by $\mathcal{F}(U)$. Let $\mu, v \in \mathcal{F}(U)$ and $x \in U$. Then the union and intersection of $\mu$ and $v$ are defined in the following way:

$$
\begin{aligned}
& (\mu \vee v)(x)=\mu(x) \vee v(x), \\
& (\mu \wedge v)(x)=\mu(x) \wedge v(x),
\end{aligned}
$$

$\mu \leq v$ if and only if $\mu(x) \leq v(x)$ for all $x \in U$.

Definition 3 [4] Let $U$ be a common universe, $E$ be a set of parameters and $A \subseteq E$. Then a pair $(F, A)$ is called a fuzzy soft set over $U$, where $F$ is a mapping given by $F: A \rightarrow \mathcal{F}(U)$.

Definition 4 [4] For two fuzzy soft sets $(F, A)$ and $(G, B)$ over a common universe $U$, we say that $(F, A)$ is a fuzzy soft subset of $(G, B)$ if

(i) $A \subseteq B$,

(ii) $F(a) \leq G(a)$ for all $a \in A$.

In this case, we write $(F, A) \widetilde{\subseteq}(G, B)$.

Definition 5 [14] The relative complement of a fuzzy soft set $(F, A)$ is denoted by $(F, A)^{c}$ and is defined by $(F, A)^{c}=\left(F^{c}, A\right)$, where $F^{c}: A \rightarrow \mathcal{F}(U)$ is a mapping given by $F^{c}(a)=$ $1-F(a)$ for all $a \in A$.

It should be noted that $1-F(a)$ denotes the fuzzy complement of $F(a)$.

\section{Definition 6 [4]}

(i) A fuzzy soft set $(F, A)$ is said to be the absolute fuzzy soft set over $U$, denoted by $\Omega$, if $F(a)=1_{U}$ for all $a \in A$.

(ii) A fuzzy soft set $(F, A)$ is said to be the null fuzzy soft set over $U$, denoted by $\Phi$, if $F(a)=0_{U}$ for all $a \in A$.

Definition $7[4,14]$ Let $(F, A)$ and $(G, B)$ be two fuzzy soft sets over a common universe $U$. Then 
(i) The union of fuzzy soft sets $(F, A)$ and $(G, B)$ is defined as the fuzzy soft set $(H, C)=(F, A) \widetilde{\cup}(G, B)$ over $U$, where $C=A \cup B$ and

$$
H(c)= \begin{cases}F(c) & \text { if } c \in A \backslash B, \\ G(c) & \text { if } c \in B \backslash A, \\ F(c) \vee G(c) & \text { if } c \in A \cap B\end{cases}
$$

for all $c \in C$.

(ii) The restricted intersection of fuzzy soft sets $(F, A)$ and $(G, B)$ is defined as the fuzzy soft set $(H, C)=(F, A) \widetilde{\sqcap}(G, B)$ over $U$, where $C=A \cap B \neq \emptyset$ and $H(c)=F(c) \wedge G(c)$ for all $c \in C$.

(iii) The restricted union of fuzzy soft sets $(F, A)$ and $(G, B)$ is defined as the fuzzy soft set $(H, C)=(F, A) \widetilde{\sqcup}(G, B)$ over $U$, where $C=A \cap B \neq \emptyset$ and $H(c)=F(c) \vee G(c)$ for all $c \in C$.

(iv) The extended intersection of fuzzy soft sets $(F, A)$ and $(G, B)$ is defined as the fuzzy soft set $(H, C)=(F, A) \widetilde{\cap}(G, B)$ over $U$, where $C=A \cup B$ and

$$
H(c)= \begin{cases}F(c) & \text { if } c \in A \backslash B, \\ G(c) & \text { if } c \in B \backslash A, \\ F(c) \wedge G(c) & \text { if } c \in A \cap B\end{cases}
$$

for all $c \in C$.

(v) The $\wedge$-intersection of fuzzy soft sets $(F, A)$ and $(G, B)$ is defined as the fuzzy soft set $(H, C)=(F, A) \widetilde{\wedge}(G, B)$ over $U$, where $C=A \times B$ and $H(a, b)=F(a) \wedge G(b)$ for all $(a, b) \in A \times B$.

(vi) The $\vee$-union of fuzzy soft sets $(F, A)$ and $(G, B)$ is defined as the fuzzy soft set $(H, C)=(F, A) \widetilde{\vee}(G, B)$ over $U$, where $C=A \times B$ and $H(a, b)=F(a) \vee G(b)$ for all $(a, b) \in A \times B$.

Example Let $U=\left\{h_{1}, h_{2}, h_{3}\right\}$ be the set of three houses under consideration and $E=$ $\left\{e_{1}\right.$ (costly), $e_{2}$ (beautiful), $e_{3}$ (green surroundings) $\}$ be the set of parameters.

Consider two fuzzy soft sets $(F, A)$ and $(G, B)$, where $A=\left\{e_{1}, e_{2}\right\}$ and $B=\left\{e_{1}, e_{2}, e_{3}\right\}$ given by $(F, A)=\left\{F\left(e_{1}\right)=\left\{\left(h_{1}, .7\right),\left(h_{2}, .5\right),\left(h_{3}, .3\right)\right\}, F\left(e_{2}\right)=\left\{\left(h_{1}, .7\right),\left(h_{2}, .6\right),\left(h_{3}, .5\right)\right\}\right\}$ and $(G, B)=$ $\left\{G\left(e_{1}\right)=\left\{\left(h_{1}, .7\right),\left(h_{2}, .5\right),\left(h_{3}, .3\right)\right\}, G\left(e_{2}\right)=\left\{\left(h_{1}, .7\right),\left(h_{2}, .6\right),\left(h_{3}, .5\right)\right\}, G\left(e_{3}\right)=\left\{\left(h_{1}, .2\right),\left(h_{2}, .4\right)\right.\right.$, $\left.\left.\left(h_{3}, .5\right)\right\}\right\}$. Then

(i) $(F, A)^{c}=F^{c}\left(e_{1}\right)=\left\{\left(h_{1}, .3\right),\left(h_{2}, .5\right),\left(h_{3}, .7\right)\right\}, F^{c}\left(e_{2}\right)=\left\{\left(h_{1}, .3\right),\left(h_{2}, .4\right),\left(h_{3}, .5\right)\right\}$,

(ii) $(F, A) \widetilde{\widetilde{C}}(G, B)$.

Proposition $[4,14]$ Let $(F, A)$ and $(G, B)$ be two fuzzy soft sets over a common universe $U$. Then

(1) $(F, A) \widetilde{\cup}(F, A)=(F, A)$,

(2) $(F, A) \widetilde{\cap}(F, A)=(F, A)$,

(3) $((F, A) \widetilde{\cup}(G, B))^{c}=(F, A)^{c} \widetilde{\cap}(G, B)^{c}$,

(4) $((F, A) \widetilde{\cap}(G, B))^{c}=(F, A)^{c} \widetilde{\cup}(G, B)^{c}$,

(5) $((F, A) \widetilde{\nabla}(G, B))^{c}=(F, A)^{c} \widetilde{\wedge}(G, B)^{c}$,

(6) $((F, A) \widetilde{\wedge}(G, B))^{c}=(F, A)^{c} \widetilde{\nabla}(G, B)^{c}$. 


\section{Definition 8 [12]}

(1) A fuzzy subset $\mu$ on the universe of discourse $\mathbb{R}$ (the set of all real numbers) is convex if and only if for $a, b \in U \mu(\alpha a+\beta b) \geq \mu(a) \wedge \mu(b)$, where $\alpha+\beta=1$.

(2) A fuzzy subset $\mu$ on the universe of discourse $U$ is called a normal fuzzy subset if there exist $a_{i} \in U$ such that $\mu\left(a_{i}\right)=1$.

(3) A fuzzy number is a fuzzy subset defined on the universe of discourse $\mathbb{R}$ which is both convex and normal.

A fuzzy number $\mu$ on the universe of discourse $\mathbb{R}$ may be characterized by a triangular distribution function parameterized by a triplet $(a, b, c)$. The membership function of the fuzzy number $\mu$ is defined as

$$
\mu(u)= \begin{cases}0 & \text { if } u<a, \\ \frac{u-a}{b-a} & \text { if } a \leq u \leq b, \\ \frac{c-u}{c-b} & \text { if } b \leq u \leq c, \\ 0 & \text { if } u>c .\end{cases}
$$

If the membership function $\mu(u)$ is piecewise linear, then $\mu$ is said to be a trapezoidal fuzzy number.

Let $\mu$ and $\beta$ be two triangular fuzzy numbers parameterized by the triplet $b_{1}=\left(a_{1}, b_{1}, c_{1}\right)$ and $b_{2}=\left(a_{2}, b_{2}, c_{2}\right)$ respectively. Then addition and multiplication of $\mu$ and $\beta$ as given in $[12]$ are

$$
\begin{aligned}
\mu \oplus \beta & =\tilde{a}_{2} \oplus \widetilde{b}_{2}=\left(a_{1}, a_{2}, a_{3}\right) \oplus\left(b_{1}, b_{2}, b_{3}\right) \\
& =\left(a_{1}+b_{1}, a_{2}+b_{2}, a_{3}+b_{3}\right)
\end{aligned}
$$

and

$$
\begin{aligned}
\mu \otimes \beta & =\tilde{a}_{2} \otimes \widetilde{b}_{2}=\left(a_{1}, a_{2}, a_{3}\right) \otimes\left(b_{1}, b_{2}, b_{3}\right) \\
& =\left(a_{1} \times b_{1}, a_{2} \times b_{2}, a_{3} \times b_{3}\right) .
\end{aligned}
$$

Next we give the defuzzification method of a trapezoidal fuzzy number. Take a trapezoidal fuzzy number parameterized by a quadruplet $(p, q, r, s)$ as shown in Figure 1 .

Then the defuzzification value $t$ of the fuzzy number is calculated from the figure as follows:

$$
\begin{aligned}
& (t-q)(1)+\frac{1}{2}(q-p)(1)=(r-t)(1)+\frac{1}{2}(s-r)(1) \\
& \Rightarrow \quad(t-q)+\frac{1}{2}(q-p)=(r-t)+\frac{1}{2}(s-r) \\
& \Rightarrow \quad 2 t=\frac{s-r-q+p}{2}+q+r \\
& \Rightarrow \quad 2 t=\frac{p+q+r+s}{2} \\
& \Rightarrow \quad t=\frac{p+q+r+s}{4} .
\end{aligned}
$$




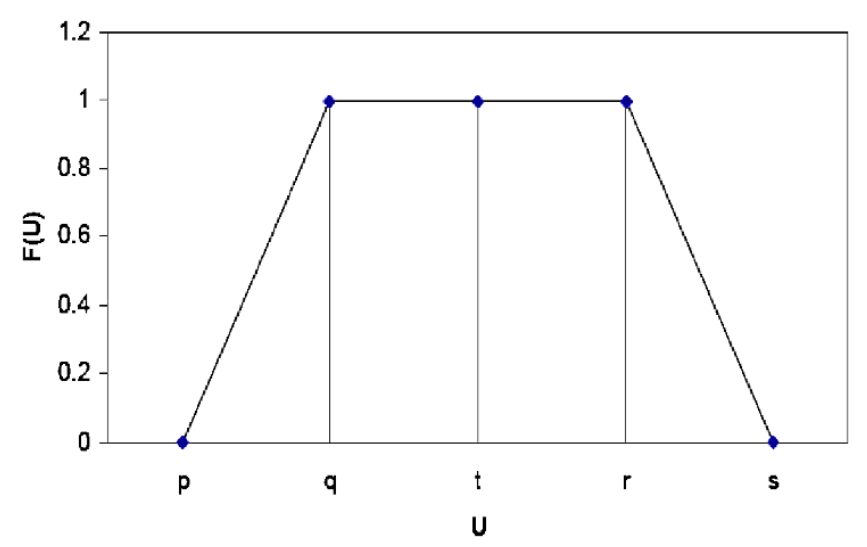

Figure 1 A trapezoidal fuzzy number $M=(p, q, r, s)$. A trapezoidal fuzzy number parameterized by a quadruplet $(p, q, r, s)$

Similarly, the defuzzification value $e$ of a triangular fuzzy number $(a, b, c)$ is equal to

$$
e=\frac{a+b+b+c}{4}
$$

\section{Methodology and algorithm}

In this section we present an algorithm for medical diagnosis using fuzzy arithmetic operations. Assume that there is a set of $m$ patients, $P=\left\{p_{1}, p_{2}, p_{3}, \ldots, p_{m}\right\}$ with a set of $n$ symptoms $S=\left\{s_{1}, s_{2}, s_{3}, \ldots, s_{n}\right\}$ related to a set of $k$ diseases $D=\left\{d_{1}, d_{2}, d_{3}, \ldots, d_{k}\right\}$.

We apply fuzzy soft set theory to develop a technique through Sanchez's method to diagnose which patient is suffering from what disease. For this, construct a fuzzy soft set $(F, P)$ over $S$ where $F$ is a mapping $F: P \rightarrow \mathcal{F}(S)$. This fuzzy soft set gives a relation matrix $Q$, called patient-symptom matrix, where the entries are fuzzy numbers $\widetilde{p}$ parameterized by a triplet $(p-1, p, p+1)$.

Then construct another fuzzy soft set $(G, S)$ over $D$, where $G$ is a mapping $G: S \rightarrow \mathcal{F}(D)$. This fuzzy soft set gives a relation matrix (weighted matrix) $R$, called symptom-disease matrix, where each element denotes the weight of the symptoms for a certain disease. These elements are also taken as triangular fuzzy numbers.

Thus the general form of $Q$ is

$$
Q=\begin{gathered}
p_{1} \\
p_{2} \\
p_{3} \\
\cdot \\
\cdot \\
\cdot \\
p_{m}
\end{gathered}\left[\begin{array}{ccccccc}
s_{1} & s_{2} & s_{3} & \cdot & \cdot & \cdot & s_{n} \\
\tilde{a}_{11} & \tilde{a}_{12} & \tilde{a}_{13} & \cdot & \cdot & \cdot & \tilde{a}_{1 n} \\
\tilde{a}_{21} & \tilde{a}_{22} & \tilde{a}_{23} & \cdot & \cdot & \cdot & \tilde{a}_{2 n} \\
\tilde{a}_{31} & \tilde{a}_{32} & \tilde{a}_{33} & \cdot & \cdot & \cdot & \tilde{a}_{3 n} \\
\cdot & \cdot & \cdot & \cdot & \cdot & \cdot & \cdot \\
\cdot & \cdot & \cdot & \cdot & \cdot & \cdot & \cdot \\
\cdot & \cdot & \cdot & \cdot & \cdot & \cdot & \cdot \\
\tilde{a}_{m 1} & \tilde{a}_{m 2} & \tilde{a}_{m 3} & \cdot & \cdot & \cdot & \tilde{a}_{m n}
\end{array}\right]
$$


and the general form of $R$ is

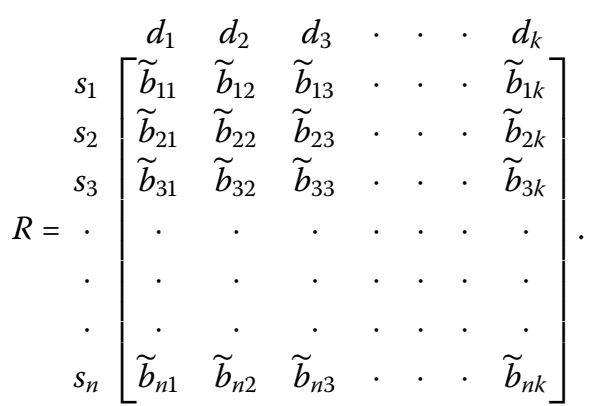

Now, performing the transformation operation $Q \otimes R$, we get the patient-diagnosis matrix $D^{*}$ as follows:

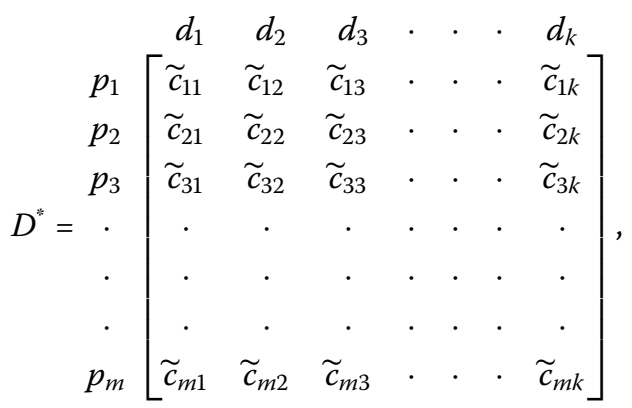

where

$$
\widetilde{c}_{i l}=\left(\sum_{j=1}^{n}\left(a_{i j}-1\right) \cdot\left(b_{j l}-1\right), \sum_{j=1}^{n} a_{i j} \cdot b_{j l}, \sum_{j=1}^{n}\left(a_{i j}+1\right) \cdot\left(b_{j l}+1\right)\right) .
$$

Then, defuzzifying each element of the above matrix by (1), we get the crisp diagnosis matrix as

$$
\begin{aligned}
& \begin{array}{lllllll}
d_{1} & d_{2} & d_{3} & \cdot & \cdot & \cdot & d_{k}
\end{array} \\
& p_{1}\left[\begin{array}{lllllll}
\widetilde{v}_{11} & \widetilde{v}_{12} & \widetilde{v}_{13} & \cdot & \cdot & \cdot & \widetilde{v}_{1 k} \\
\tilde{v}_{21} & \widetilde{v}_{22} & \widetilde{v}_{23} & \cdot & \cdot & \widetilde{v}_{2 k}
\end{array}\right] \\
& \begin{array}{llllllll}
p_{2} & \tilde{v}_{21} & \tilde{v}_{22} & \widetilde{v}_{23} & \cdot & \cdot & \cdot & \widetilde{v}_{2 k}
\end{array} \\
& \begin{array}{llllllll}
p_{3} & \widetilde{v}_{31} & \widetilde{v}_{32} & \widetilde{v}_{33} & \cdot & \cdot & \cdot & \widetilde{v}_{3 k}
\end{array}
\end{aligned}
$$

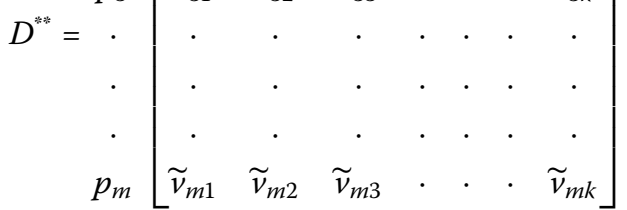

Now if $\max v_{i l}=v_{i s}$ for $1 \leq l \leq k$, then we conclude that the patient $p_{i}$ is suffering from disease $d_{s}$. In case max $v_{i l}$ occurs for more than one value of $l, 1 \leq l \leq m$, then we can reassess the symptoms to break the tie.

\section{Algorithm}

Step I: Input the soft set $(F, P)$ to obtain the patient-symptom matrix $Q$.

Step II: Input the soft set $(G, S)$ to obtain the symptom-disease matrix $R$. 
Step III: Perform the transformation operation $Q \otimes R$ to get the patient diagnosis matrix $D^{\prime \prime}$.

Step IV: Defuzzify all the elements of the matrix $D^{\prime \prime}$ by (1) to obtain the matrix $D^{m * n}$.

Step V: Find $s$ for which $v_{i s}=\max v_{i l}$.

Then we conclude that the patient $p_{i}$ is suffering from disease $d_{s}$.

\section{Case study}

Suppose there are three patients John, George and Albert in a hospital with symptoms temperature, headache, cough and stomach problem. Let the possible diseases relating to the above symptoms be viral fever, typhoid and malaria. Now take $P=\left\{p_{1}, p_{2}, p_{3}\right\}$ as the universal set where $p_{1}, p_{2}$ and $p_{3}$ represent patients John, George and Albert, respectively. Next consider the set $S=\left\{s_{1}, s_{2}, s_{3}, s_{4}\right\}$ as a universal set where $s_{1}, s_{2}, s_{3}, s_{4}$ represent symptoms temperature, headache, cough and stomach problem, respectively and the set $D=\left\{d_{1}, d_{2}, d_{3}\right\}$, where $d_{1}, d_{2}$ and $d_{3}$ represent the diseases viral fever, typhoid and malaria, respectively.

Suppose

$$
\begin{aligned}
& F\left(p_{1}\right)=\left\{s_{1} / \widetilde{7}, s_{2} / \widetilde{3}, s_{3} / \widetilde{5}, s_{4} / \widetilde{2}\right\}, \quad F\left(p_{2}\right)=\left\{s_{1} / \widetilde{6}, s_{2} / \widetilde{2}, s_{3} / \widetilde{3}, s_{4} / \widetilde{5}\right\} \\
& F\left(p_{3}\right)=\left\{s_{1} / \widetilde{3}, s_{2} / \widetilde{5}, s_{3} / \widetilde{3}, s_{4} / \widetilde{6}\right\} .
\end{aligned}
$$

Then the fuzzy soft set $(F, P)$ is a parameterized family of all fuzzy sets over $S$ and gives a collection of an approximate description of the patient-symptoms in the hospital. This fuzzy soft set $(F, P)$ represents the relation matrix (patient-symptom matrix) $Q$ and is given by

$$
Q=\begin{aligned}
& p_{1} \\
& p_{2} \\
& p_{3}
\end{aligned}\left[\begin{array}{cccc}
\widetilde{7} & s_{2} & s_{3} & s_{4} \\
\widetilde{6} & \widetilde{5} & \widetilde{2} & \widetilde{3} \\
\widetilde{3} & \widetilde{5} & \widetilde{3} & \widetilde{6}
\end{array}\right] .
$$

Next suppose

$$
\begin{array}{ll}
G\left(s_{1}\right)=\left\{d_{1} / \widetilde{9}, d_{2} / \widetilde{5}, d_{3} / \widetilde{1}\right\}, & G\left(s_{2}\right)=\left\{d_{1} / \widetilde{3}, d_{2} / \widetilde{5}, d_{3} / \widetilde{5}\right\}, \\
G\left(s_{3}\right)=\left\{d_{1} / \widetilde{5}, d_{2} / \widetilde{2}, d_{3} / \widetilde{5}\right\}, & G\left(s_{4}\right)=\left\{d_{1} / \widetilde{2}, d_{2} / \widetilde{8}, d_{3} / \widetilde{8}\right\} .
\end{array}
$$

Then the fuzzy soft set $(G, S)$ is a parameterized family $\left\{G\left(s_{1}\right), G\left(s_{2}\right), G\left(s_{3}\right), G\left(s_{4}\right)\right\}$ of all fuzzy sets over the set $S$ where $G: S \rightarrow \mathcal{F}(D)$ and is determined from expert medical documentation. Thus the fuzzy soft set $(G, S)$ gives an approximate description of the three diseases and their symptoms. This soft set is represented by a relation matrix (symptomdisease matrix) $R$ and is given by

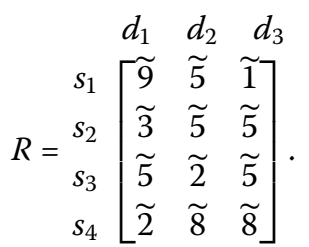


Then, performing the transformation operation $Q \otimes R$, we get the patient-diagnosis matrix $D^{\prime \prime}$ as

$$
D^{*}=p_{1} p_{2}\left[\begin{array}{ccc}
d_{1} & d_{2} & d_{3} \\
p_{3} & \widetilde{76} & \widetilde{63} \\
\widetilde{85} & \widetilde{86} & \widetilde{71} \\
\widetilde{69} & \widetilde{94} & \widetilde{91}
\end{array}\right]
$$

where

$$
\begin{array}{lll}
\widetilde{101}=(69,101,141), & \widetilde{76}=(44,76,117), & \widetilde{63}=(31,63,103), \\
\widetilde{85}=(54,85,124), & \widetilde{86}=(54,86,126), & \widetilde{71}=(40,71,101), \\
\widetilde{69}=(37,69,109), & \widetilde{94}=(61,94,135), & \widetilde{91}=(59,91,131) .
\end{array}
$$

The above values are obtained from $(*)$.

Now, defuzzifying the above matrix, we get

$$
D^{* * *}=p_{1} p_{2}\left[\begin{array}{ccc}
d_{1} & d_{2} & d_{3} \\
p_{3}
\end{array}\left[\begin{array}{ccc}
103 & 78.25 & 65 \\
87 & 88 & 70.75 \\
71 & 96 & 93
\end{array}\right] .\right.
$$

It is clear from the above matrix that patient $p_{1}$ is suffering from disease $d_{1}$ and patients $p_{2}$ and $p_{3}$ both are suffering from disease $d_{2}$.

\section{Conclusion}

We have applied the notion of fuzzy soft sets in Sanchez's method of medical diagnosis. A case study has been taken to exhibit the simplicity of the technique. Future work in this regard would be required to study whether the notions put forward in this paper yield a fruitful result.

Competing interests

The authors declare that they have no competing interests.

Authors' contributions

The present study was proposed by YÇ and SY. All authors read and approved the final manuscript.

\section{Acknowledgements}

Dedicated to Professor Hari M Srivastava.

Received: 26 November 2012 Accepted: 14 February 2013 Published: 1 March 2013

\section{References}

1. Zadeh, LA: Fuzzy sets. Inf. Control 8, 338-353 (1965)

2. Pawlak, Z: Rough sets. Int. J. Comput. Inf. Sci. 11, 341-356 (1982)

3. Molodtsov, D: Soft set theory - first result. Comput. Math. Appl. 37, 19-31 (1999)

4. Maji, PK, Biswas, R, Roy, AR: Fuzzy soft set. J. Fuzzy Math. 9(3), 677-692 (2001)

5. Neog, TJ, Sut, DK: Theory of fuzzy soft sets from a new perspective. Int. J. Latest Trends Comput. 2(3), $439-450$ (2011)

6. De, SK, Biswas, R, Roy, AR: An application of intuitionistic fuzzy sets in medical diagnosis. Fuzzy Sets Syst. 117, 209-213 (2001)

7. Sanchez, E: Resolution of composite fuzzy relation equations. Inf. Control 30, 38-48 (1976)

8. Sanchez, E: Inverse of fuzzy relations, application to possibility distributions and medical diagnosis. Fuzzy Sets Syst. 2(1), 75-86 (1979) 
9. Saikia, BK, Das, PK, Borkakati, AK: An application of intuitionistic fuzzy soft sets in medical diagnosis. Bio-Sci. Res. Bull. 19(2), 121-127 (2003)

10. Chetia, B, Das, PK: An application of interval valued fuzzy soft set in medical diagnosis. Int. J. Contemp. Math. Sci. 5(38), 1887-1894 (2010)

11. Meenakshi, AR, Kaliraja, M: An application of interval valued fuzzy matrices in medical diagnosis. Int. J. Math. Anal. 5(36), 1791-1802 (2011)

12. Kaufmann, A, Gupta, MM: Introduction to Fuzzy Arithmetic Theory and Applications. Van Nostrand-Reinhold, New York (1991)

13. Ali, MI, Feng, F, Liu, X, Min, WK, Shabir, M: On some new operations in soft set theory. Comput. Math. Appl. 57, 1547-1553 (2009)

14. Ali, Ml, Shabir, M: Comments on De Morgan's law in fuzzy soft sets. J. Fuzzy Math. 18(3), 679-686 (2010)

doi:10.1186/1029-242X-2013-82

Cite this article as: Celik and Yamak: Fuzzy soft set theory applied to medical diagnosis using fuzzy arithmetic operations. Journal of Inequalities and Applications 2013 2013:82.

Submit your manuscript to a SpringerOpen ${ }^{\circ}$ journal and benefit from:

- Convenient online submission

- Rigorous peer review

- Immediate publication on acceptance

- Open access: articles freely available online

- High visibility within the field

- Retaining the copyright to your article 